\title{
Niosomes: a promising drug delivery system
}

\author{
Valeria Shakhova ${ }^{1}$, Valery Belyaev ${ }^{1}$, Elena Kastarnova $^{1, *}$, Vladimir Orobets ${ }^{1}$, Elena \\ Grudeva $^{1}$ \\ ${ }^{1}$ The Stavropol State Agrarian University, 12, Zootechnichesky lane, 357017, Stavropol, Russia
}

\begin{abstract}
Targeting is a phenomenon in which the distribution of a drug in the body occurs in such a way that the main part of it interacts with the target tissue at the cellular or subcellular level to achieve the desired pharmacological effect on the selected site without undesirable interactions in other organs. This can be achieved using a drug delivery system such as niosomes, which are non-ionic vesicles of surfactants obtained by hydrating synthetic non-ionic surfactants with the inclusion of cholesterol. They are vestibular systems similar to liposomes that can be used as carriers of amphiphilic or lipophilic drugs. Niosomes are a promising drug delivery tool, and it has been widely evaluated as a possibility of controlled release and targeted delivery of the active substance for the treatment of cancer, autoimmune diseases, viral and other infectious diseases. It can be assumed that encapsulation of the drug in the vesicular system prolongs its presence in the systemic circulation and increases the possibility of penetration into the target tissue, possibly reducing toxicity if selective absorption can be achieved.
\end{abstract}

\section{Introduction}

Niosomes are vesicles based on nonionic surfactants. They were originally developed as an alternative system for controlled drug delivery to liposomes to overcome problems related to sterilization, large-scale production and stability $[1,2,3]$.

Hydration of the membrane, which includes a mixture of single or double alkyl chain and cholesterol, leads to the formation of vesicular dispersions. These dispersions were called niosomes [5]. In fact, these vesicles do not form spontaneously. Thermodynamically stable vesicles are formed only in the presence of mixtures of surfactants and a stabilizing membrane $[1,2,4]$.

For the first time, niosomes were used as a delivery system for anti-cancer drugs $[1,2]$. They had the ability to alter the pharmacokinetic profile, distribution within the organ, and metabolism of methotrexate in mice [3].

Niosomes are versatile in structure, morphology, and size; they can capture hydrophilic drugs in an aqueous medium or lipophilic drugs by dividing these molecules into two-layer domains. In addition, they can be designed as single-layer, oligolamellar or multi-layer. Niosomes are also highly stable, cost-effective, and allow for large-scale production $[1,3]$.

Niosomes consist of two main components, such as cholesterol and non-ionic

*Corresponding author: elena-kastarnova@mail.ru 
surfactants. Cholesterol provides stability and the appropriate form. Surfactants take part in an important function of niosomes formation. Surfactants of nonionic groups have different classes in the form of Span 20, Span 40, Span 60, Span 80 and Span 85. Also, the surfactant Tween has different names: Tween 20, Tween 40, Tween 60 and Tween 80. Brij surfactant has many types: Brij 30, Brij 35, Brij 52, Brij 58, Brij 72 и Brij 76.

These surfactants are usually used for niosomes production. Other surfactants that form niosomes are: etheric bound surfactants; surfactants with an alkyl chain; sorbitan esters; polysorbates.

Unlike naturally occurring phospholipids (the main liposomal lipids), which have double alkyl chains, the most widely used surfactants that form niosomes are singlestranded, synthetic, nonionic surfactants [4]. Alkyl esters and alkaloids, as well as compounds of fatty acids and amino acids can form vesicles [5]. Recently, niosomes composed of L64 and P105 (copolymers of ethylene oxide and propylene oxide) have been prepared and used as a transdermal delivery system for sodium sulfadiazine (a model hydrophilic drug) [3].

Alkyl glycerol esters, especially hexadecyldiglycerol ether (C16G2), have been described as niosome-forming surfactants and have been used for drug delivery, such as experimental cancer chemotherapy [6].

These surfactants have been used to alter the pharmacokinetics of methotrexate and doxorubicin. Alkyl glycerol esters have also been used to encapsulate sodium stibogluconate to fight leishmaniasis [6,7].

The second group of alkyl ether surfactants, in which the hydrophilic region consists of repeated oxyethylene units, was used to encapsulate insulin during oral delivery to prevent its inactivation by gastric juice [8].

Alkyl esters, such as sorbitan and fatty acid esters (Span) and polyoxyethylene sorbitan and fatty acid esters (Tween), are widely used in cosmetics and food products, as well as in modern pharmaceuticals. They are considered non-toxic and non-irritating materials [9]. These surfactants were used in the production of the niosomes as a drug as a delivery system, including oral, transdermal and ophthalmic drug delivery. Studies of cell culture toxicity have shown that niosomes consisting of esters of surfactants are less toxic than ether-type niosomes. This can be attributed to the possible enzymatic cleavage of ester bonds $[7,8]$.

Some water-soluble surfactants, such as Tween 20 and Tween 61 , cannot form vesicles under normal conditions. These surfactants can form vesicles when mixed with cholesterol. Due to its molecular shape and solubility, it fills the empty spaces among the amphiphiles, thereby fixing them stronger in the two-layer structure [6].

It was found that cholesterol in niosomes increases the stability of the membrane, reduces the fluidity, permeability, and changes in the membrane [9].

Other excipients, membrane additives, such as charge inductors, play a vital function for the niosomes. Their role is to increase the surface charge density, prevent vesicle flocculation, aggregation, and fusion. There are negatively and positively charged molecules $[3,6,9]$. Examples of charge inductors are:

1. Dicetylphosphate (DCP) - negative charge

2. Stearylamine (SA) - positive charge.

The size of particles in the niosomes determines their behavior in the body. Particles smaller than $100 \mathrm{~nm}$ have been reported to easily penetrate tumor tissue. This is due to the fact that nanoparticles can penetrate through the capillaries is intermittent and tumor tissues fenestra. The size reduction step is sometimes included in the niosome production procedure, after the initial hydration stage. This is done in order to improve the biodistribution of systemically injected niosomes and to target the injected niosomes to the affected area $[2,5,6,7,9]$. 
The main method of noisome creating involves the use of organic solvents during evaporation of which a lipid membrane is formed with subsequent hydration of the aqueous medium. However, there are different methods (table 1) [10-15].

Table 1. The methods of noisome creating.

\begin{tabular}{|c|c|}
\hline \multicolumn{2}{|c|}{ Small Single-Layer Vesicles } \\
\hline \multicolumn{2}{|c|}{ Methods } \\
\hline Mltrasound Exposure & Microfluidization \\
\hline \multicolumn{2}{|c|}{ Multilayer Vesicles } \\
\hline Manual Shaking & Transmembrane Ph Gradient \\
\hline Large Single-Layer Vesicles \\
\hline \multicolumn{2}{|c|}{ Methods } \\
\hline Reverse Phase Evaporation Technology \\
\hline
\end{tabular}

\section{The creation of small single-layer vesicles}

1. Ultrasound exposure is a common technique for preparing niosomal vesicles. In a 10-ml glass tube containing the drug, surfactants and cholesterol are mixed with a buffer. After that, the mixture is subjected to ultrasonic treatment for 3 minutes using a titanium probe to produce niosomes. The resulting product contains small and single-layer vesicles. This method is most widely used in the preparation of small vesicles. The ultrasonic treatment process consists of two types, depending on the requirements for which it is necessary to use.

2. Microfluidization. This method contributes to the production of homogeneous and small vesicles and has better reproducibility.

\section{The development of multilayer vesicles}

1. Manual shaking method (thin membrane hydration method) In a round-bottomed flask, chloroform or methanol is added to the ether, to which a surfactant, cholesterol, is added and mixed. The temperature is maintained around $\left(60^{\circ} \mathrm{C}\right)$ when this process is carried out, the volatile solvent is evaporated, which leads to the deposition of a lipid film on the walls of the flask, after which the addition of an aqueous phase at $45{ }^{\circ} \mathrm{C}$ leads to the production of multilayer multilamellar vesicles.

2. Transmembrane $\mathrm{pH}$ gradient

The organic solvent chloroform is poured on the round bottom of the flask, to which the surfactant and cholesterol are added. An organic solvent during evaporation results in a lipid membrane on the side walls of the flask. In addition, the thin membrane formed undergoes a hydration process by vortex mixing with $300 \mathrm{~mm}$ citric acid $(\mathrm{pH} \mathrm{4.0)}$. The formed multi-layered niosomes are frozen and thawed three times, followed by an ultrasound treatment process of several minutes. A solution containing $10 \mathrm{mg} / \mathrm{ml}$ of the drug is added to the aqueous solution of the liposomal suspension and then shaken. Adding dioratry phosphate $(1 \mathrm{M})$ gradually increases the $\mathrm{pH}$ to $7.0-7.2$. Then stir and heat at $60^{\circ} \mathrm{C}$ for 10 minutes until the formation of multilayer vesicles.

\section{The creation of large single-layer vesicles}

\section{Reverse phase evaporation technology}

The novelty of this system is the removal of volatile organic substances by solvent during evaporation. The mixture of ether and chloroform was dispersed with the surfactant 
and cholesterol in an equal ratio $(1: 1)$ to the solvent. The aqueous phase containing the drug is added to the above solution and the resulting two phases are treated with ultrasound for several minutes. A transparent gel is formed, which is then treated with ultrasound for several minutes with the addition of a small amount of phosphate buffer solution. Under low pressure, the organic phase is removed by evaporation. The resulting suspension is further diluted with PBS solution and heated in a water bath at an optimal temperature $\left(45^{\circ} \mathrm{C}\right)$ for 10 minutes to produce niosomes.

2. Method of ether introduction

To nonionic surfactants and cholesterol, warm water is gradually added at a temperature of $60{ }^{\circ} \mathrm{C}$. In addition, the ether containing a mixture of the medicinal solution is slowly added to the phosphate buffer. The diameter of the niosome varies in the size range from 50 to $1000 \mathrm{~nm}$, depending on the procedure used. The main drawback of this process is the existence of a small amount of ether in the suspension of vesicles, which is difficult to remove.

Drug delivery in ocular therapeutics is a challenging problem and is a subject of interest to scientists working in the multi-disciplinary areas pertaining to the eye, including chemical, biochemical, pharmaceutical, medical, clinical, and toxicological sciences [3]. The current treatment of ocular infections requires frequent topical antimicrobial drug administration in microbial keratitis and repeated injection of antimicrobial drugs into the site of infection in endophthalmitis [4]. The therapeutics requires the association of more than one antimicrobial treatment for several weeks, which often leads to poor patient compliance, contributing to low therapy efficiency [5]. In order to overcome the problems of conventional ocular therapy, such as short residence time, loss of drug through nasolacrimal drainage, impermeability of corneal epithelium and frequent instillation; newer ocular delivery systems for gentamicin are being explored by many researchers [68]. It is now common knowledge that topical controlled delivery of ophthalmic drugs improves their ocular bioavailability with respect to traditional eye drops, by decreasing the rate of drug elimination from the precorneal area $[9,17]$.

The advantage of vesicular systems does not only reside in providing prolonged and controlled action at the corneal surface but also involves providing controlled ocular delivery by preventing the metabolism of the drug from the enzymes present at the tear/corneal epithelial surface. Moreover, vesicles offer a promising avenue to fulfill the need for an ophthalmic drug delivery system that has the convenience of a drop, but will localize and maintain drug activity at its site of action. The penetration of drug molecules into the eye from a topically applied preparation is a complex phenomenon. In vesicular dosage forms, the drug is encapsulated in lipid vesicles, which can cross cell membrane. Vesicles, therefore, can be viewed as drug carriers which can change the rate and extent of absorption as well as the disposition of the drug.

Vesicular drug delivery systems used in ophthalmics broadly include niosomes [3, 17].

Niosomes in topical ocular delivery are preferred over other vesicular systems because of the following reasons: [1] chemical stability; [2] low toxicity because of their non-ionic nature; [3] handling surfactants with no special precautions or conditions; [4] the ability to improve the performance of the drug via better availability and controlled delivery at a particular site; [5] being biodegradable, biocompatible and non-immunogenic [12].

Various studies have demonstrated the successful use of niosomes as ocular drug delivery carriers where these vesicles significantly improved the ocular bioavailability of cyclopentolate, with respect to reference buffer solution. No irritation with the niosomal formulation was observed [13]. Reported that there was approximately a 2.5 times increase in the ocular bioavailability of timolol maleate (a water soluble drug) encapsulated in niosomes as compared to timolol maleate solution. An increased ocular bioavailability of water-soluble drugs, entrapped in niosomes, may be due to the fact that surfactants act as 
penetration enhancers by removing the mucus layer and breaking junctional complexes [15-17].

The effectiveness of including the drug in the vesicles may depend on the small internal volume and leakage of the drug from single-layer vesicles of small and medium size (up to $100 \mathrm{~nm})[1,6]$. Reduction in the size of vesicles can be achieved by a number of methods:

- Probe treatment with ultrasound can lead to the formation of niosomal vesicles ranging in size from 100 to $140 \mathrm{~nm}$.

- Extrusion through polycarbonate filters using liposomal extruders.

- A combination of ultrasound treatment and filtration was used to prepare doxorubicin. Niosomes in the size range of $200 \mathrm{~nm}$.

- High pressure homogenization also results in the formation of vesicles with a diameter of less than $100 \mathrm{~nm}$.

Drugs included in niosome vesicles: for intravenous administration: doxorubicin, methotrexate, tretinoin, vincristine; in ophthalmology: gentamicin, ofloxacin, timolola maleate, cyclopentalate; nasal administration: sumatriptan, viral flu vaccine; transdermal administration: fluriprofen, piroxicam, ketrolac.

Many studies were performed on liposomal and niosomal vesicles as therapeutic drug carrier systems to reduce the drug toxicity by alternating drug pharmacokinetics or modifying the drug delivery in order to prolong drug action at the target site. The antibiotics-loaded vesicles showed enhanced drug concentrations at the site of action due to targeting of drug to the infected tissues, increase in the intracellular antibiotic concentrations, and reduced the toxicities of potentially toxic antibiotics resulting from the targeting of antimicrobial drugs to the infectious organism.

Niosomes systems might be capable of ensuring different pathways of interaction with microbial cells, compared to entering of fluoroquinolones in to cells from other dosage forms. This behavior may be useful in the treatment of infections caused by quinoloneresistant bacteria or by microbes which are, normally, poorly sensitive to this class of drugs. So, the entrapment of the fluoroquinolones in niosomes could be of therapeutic interest and could improve the efficacy of these drugs. Ofloxacin, second generation fluoroquinolone antibiotic used for the treatment of bacterial conjunctivitis caused by susceptible organisms has been chosen to encapsulate in the niosomes to improve the efficacy by altering the pharmacokinetics [16].

\section{Conclusion}

From the foregoing, it can be stated that the characteristics and capabilities of the niosomes are best for targeted delivery. Niosomes have been successfully used for the treatment of many diseases. Thus, niosomes represent a promising delivery system in the treatment of diseases of various origins.

\section{References}

1. D.S. Aldricha, C.M. Bacha, W. Brownb and others, Ophthalmic Preparations 39(5)

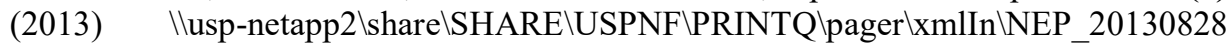
110441_S200824.xml

2. S. Nagalakshmi, N. Damodharan, J. Thanka, S. Seethalakshmi, Int. J. Pharm. Sci. Rev. Res. 32(1), 61-66 (2015)

3. R. Muzzalupo, L. Tavano, R. Cassano, S. Trombino, T. Ferrarelli, N. Picci, European Journal of Pharmaceutics and Biopharmaceutics (2011)

4. H. Abdelkader, M. Abdelkader, A thesis submitted in fulfilment of the requirements for 
the degree of Doctor of Philosophy (School of Pharmacy, The University of Auckland, 2011)

5. K.R. Prabhu, D. Koland, J. Young Pharm. 2(4), 356-361 (2010)

6. A. Sonia, P. Bharat, D. Hitesh, C. Abhishek, T. Varun, Journal of Drug Delivery \& Therapeutics 2(1), 97-99 (2012)

7. E. Moghimipour, A. Salimi, T. Yousefvand, Asian Journal of Pharmaceutics 11(3), 543-550 (2017)

8. K. Singh Rathore, R. Kumar Nema, S. Singh Sisodia, International Journal of Pharmaceutical Sciences Review and Research 23(3), 1 (2010)

9. P. Calvo, C. Remuñán-López, J. Vila-Jato, M. Alonso, Journal of Applied Polymer Science 63, 125-132 (1997)

10. K. Balagangadharan, International Journal of Biological Macromolecules 104(B), 1372-1382 (2017) doi.org/10.1016/j.ijbiomac.2016.12.046

11. M. Martínez-Martínez, European Journal of Pharmaceutics and Biopharmaceutics 136, 174-183 (2019) doi.org/10.1016/j.ejpb.2019.01.009

12. J. Park, Nanomedicine: Nanotechnology, Biology and Medicine 13(6), 2015-2025 (2017) doi.org/10.1016/j.nano.2017.04.012

13. V. Engkagul, Nanomedicine: Nanotechnology, Biology and Medicine 13(8), 25232531 (2017) doi.org/10.1016/j.nano.2017.07.001

14. L. Du, Nanomedicine: Nanotechnology, Biology and Medicine 10(7), 1411-1420 (2014) doi.org/10.1016/j.nano.2014.04.001

15. M. Naveed Yasin, S. Hussain, F. Malik, A. Hameed, T. Sultan, F. Qureshi, H. Riaz, G. Perveen, A. Wajid, Pak. J. Pharm. Sci. 25(1), 117-121 (2012)

16. Design and characterization of ofloxacin niosomes, Article in Pakistan journal of pharmaceutical sciences (2013) https://www.researchgate.net/publication/258282897

17. G. Abdelbary, AAPS PharmSciTech 9(3) (2008) DOI: 10.1208/s12249-008-9105-1 